\title{
Redesigning a Street Corridor in San Clemente, CA: South El Camino Real Urban Design Concept Plan
}

\author{
Vicente del Rio \\ PhD; Professor; City and Regional Planning Department, Cal Poly.
}

\author{
Paul Donegan, Curran K. Lord-Farmer, Jana Schwartz and Sara Steinberger \\ Master of City and Regional Planning students, Cal Poly.
}

The South El Camino Real Urban Design Concept Plan was developed by a first-year MCRP studio for the City of San Clemente, CA. The San Clemente community, the City planners and the City Council welcomed the students' ideas for making the corridor appealing, economically attractive, and safer for pedestrians and bicyclists.

In the Spring Quarter of 2015, the MCRP's first-year graduate studio was commissioned by the City of San Clemente's Planning Division to explore innovative visions for development and public investment along the South El Camino Real (SECR) corridor. The studio included 12 first-year MCRP students and an international exchange architecture senior. The work took place over an intense 10-week process in which the class went through several phases and tasks including: the analysis of existing documents, plans and legislation; a weekend-long site visit; a site assessment; a review of literature and inspirational case studies; community interviews; a project website for community comments; a comprehensive online public survey; a community meeting; a thorough analysis of constraints and opportunities for future development; visioning and preliminary concepts; and final project development.

The South El Camino Real Urban Design Concept Plan preserves the integrity of the area while introducing opportunities for development and an attractive and functional street design. The proposals enhance the pedestrian experience by promoting a safe, attractive and walkable environment, community vitality and connectivity, and increased aesthetics. The resulting corridor will serve the community while providing San Clemente with a memorable south gateway into the city.

\section{San Clemente and South El Camino Real}

Nested in Orange County's coastal hillsides midway between Los Angeles and San Diego, the City of San Clemente is home to approximately 65,000 people. Originally built as a new community by a visionary land developer in the mid 1920s as a "Spanish Village by the Sea", the city is well known for

The class included Paul Donegan, Anacany Hurtado, Sherman Kyles, Jeremy Loh, Kai Lord-Farmer, Douglas Moody, Jose Palma, Jana Schwartz, Camille Sorrant, Leana Sossikian, Sara Steinberger, and Nick Zhao (from the MCRP program); and Renato Miranda (architecture senior). The instructor was Vicente del Rio. its stunning topography, coastal landscape, scenic beaches, Spanish-style architecture, and the attractive and quaint downtown. San Clemente's location and unique qualities attract many affluent residents (including Richard Nixon who bought a vacation house there during his presidency) and a great number of tourists year around. Well known for good surfing, the city has an active surfing community, numerous surf-related businesses, and world-class surfing events.

However, while most San Clemente's neighborhoods are extremely attractive, the southern-most part of town along South El Camino Real (SECR) has an unfulfilled potential to better serve the needs and expectations of both the surrounding community and the city as a whole. The project area runs for approximately one mile along SECR (including the right-of-way and all parcels along it) between the Trestles neighborhood (east) and the San Diego Highway or l-5 (west), from Magdalena Avenue (north) to the City's southern border (open space controlled by Camp Pendleton) (Figures 1 \& 2). Along this stretch, SECR has five lanes (four for vehicular traffic, and one turning/parking lane) and a single southbound bike lane that abuts the fence and sound-wall along the I-5. There is very little space between the I-5 and the SECR corridor with a north-bound ramp leading directly into corridor at the southern edge of the city.

On the east side of the corridor, by the Trestles neighborhood, a narrow sidewalk rarely serves pedestrians who are deterred by the noisy and fast moving traffic, the sparse and unattractive commercial uses, the limited crosswalks, and the uninviting landscaping and lack of shading. While parking is plentiful and the roadway is wide, SECR is used significantly less than its full capacity and sometime serves as a diversion by motorists avoiding the I-5's heavy traffic thus worsening impacts on the local community. 


\section{The Guiding Principles: Complete Streets, Walkability and Urban Design Qualities}

Many cities across the nation face similar issues as San Clemente's SECR corridor. From the introduction of the automobile in the 1920s until recently, our built environment has been shaped to serve dispersed land uses and to accommodate vehicular traffic. The resulting cities may have streets that are easy to drive in and neighborhoods that are easy to drive through, but at the cost of placelessness and environments that are dangerous and unpleasant for people to walk or bike. This inefficient system encourages vehicular traffic in detriment of transit, cyclists and pedestrians, causing serious impacts on the quality of life and in our environment, namely air pollution and climate change.

Our project development included studying the concepts of complete streets and walkability as well as a literature search for the most appropriate urban design qualities that could support these concepts as guiding principles. In recent years, several California cities have embraced the concept of "complete streets" and "walkable" neighborhoods in plans, policies and actions; the class performed six case studies of such projects in different US cities.

Gaining traction from the mid-2000's, the concept of complete streets promotes transportation policies and the design of streets that are safe, convenient and comfortable to all modes of transportation: walking, bicycling, driving, and public transit (MacCann, Barbara \& Rynne, 2010; Smart Growth America, 2012). As of 2013, more than 490 jurisdictions in the United States had enacted complete streets policies including the State of California with the Complete Streets Act of 2008, charging CalTrans and local transportation authorities to plan for multi-modal transportation networks that meet the needs of all users no matter their level of mobility including pedestrians, bicyclists, children, seniors, persons with disabilities, motorists, and users of public transportation. Several cities and counties in California have approved specific plans and/or guidelines recognizing such concept and supporting its implementation such as San Francisco (2010) and Los Angeles County (2011).

The notion of complete streets is strongly linked to that of walkability or how much the built environment and the diversity of land uses encourage people to walk, rest, and socialize (Barnett, 2003). Thus complete streets and walkability are about improving safety, enhancing public health through walking and biking, reducing auto-dependency, encouraging social interaction, supporting community building, creating attractive environments for residents and visitors, and stimulating economic activity (NACTO, 2013; ITE, 2006).

Small and large cities throughout the US, San Clemente among them, are changing their urban policies and projects in order to accommodate the concepts of complete streets and walkability. Most of these experiences have led to significant benefits not only in quality of life and community satisfaction, but also in economic vitality. This notion is included in the Mobility \& Complete Streets Element of San Clemente's Centennial General Plan (City of San Clemente, 2014).

A literature review allowed the class to identify the eight urban design qualities most related to supporting complete streets and a walkable street environment which, according to Ewing and Bartholomew (2013) are: imageability, legibility, enclosure, human scale, transparency, linkage, complexity, and coherence. These qualities were utilized to analyze the SECR corridor during the field studies through the application of a specific analytical method developed that revealed very low walkability scores (Ewing at al 2006). The same qualities were later adopted by the class as general principles to help define the vision, objectives, and design concepts in the South EI Camino Real Urban Design Concept Plan.

\section{Public Outreach}

Since San Clemente is at least a five-hour drive from San Luis Obispo and the dynamics of the academic quarter did not allow for much travelling, the class had to maximize opportunities for public outreach in the planning process. There were four such opportunities to conduct meaningful community outreach. Firstly, during the site assessment visit students conducted surveys throughout the city ( 23 interviews) as well as in the project area and the Trestles neighborhood (30 interviews). The class also met with city planners, members of San Clemente's planning commission, and a local architect who is very active in the project area.

Secondly, the class created a website specifically dedicated to the project to inform and gather comments from the community, and utilized SurveyMonkey.com for an online survey on community perceptions and expectations for the SECR area. These tools were disseminated through a variety of outreach avenues, including the City of San Clemente's planning department, social media, and an article published in the Orange County Register. The website remained open throughout the whole quarter allowing the community to see the progress of our work and email us any concerns or ideas.

The online survey included questions about the respondent's motivations to visit (or not) the SECR area, how they normally get there, what they thought the area needed, and their preferences for street design, land uses, building types and architectural styles. A total of 128 responses were collected from May 5 th to 29th. Since the survey included three open-ended answers, their content analysis generated "word clouds" that made results more visually clear, indicating some of the respondents' perceptions and preferences.

Thirdly, San Clemente's associate planner Sean Nicolas held a special community public meeting about the project. Based on the powerpoint presentation provided by our class featuring preliminary design ideas, participants engaged in a conversation regarding potential improvements and provided several 


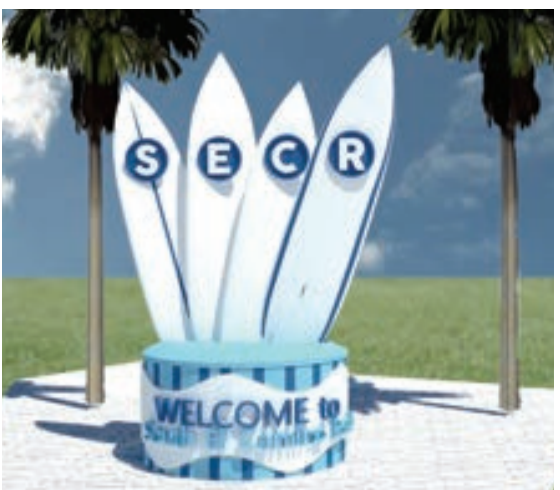

Figures 3 \& 4: The proposed landmark monument celebrates the area's surfing tradition. On the right, the proposed gateway/node in the redesigned SECR at Magdalena Avenue.

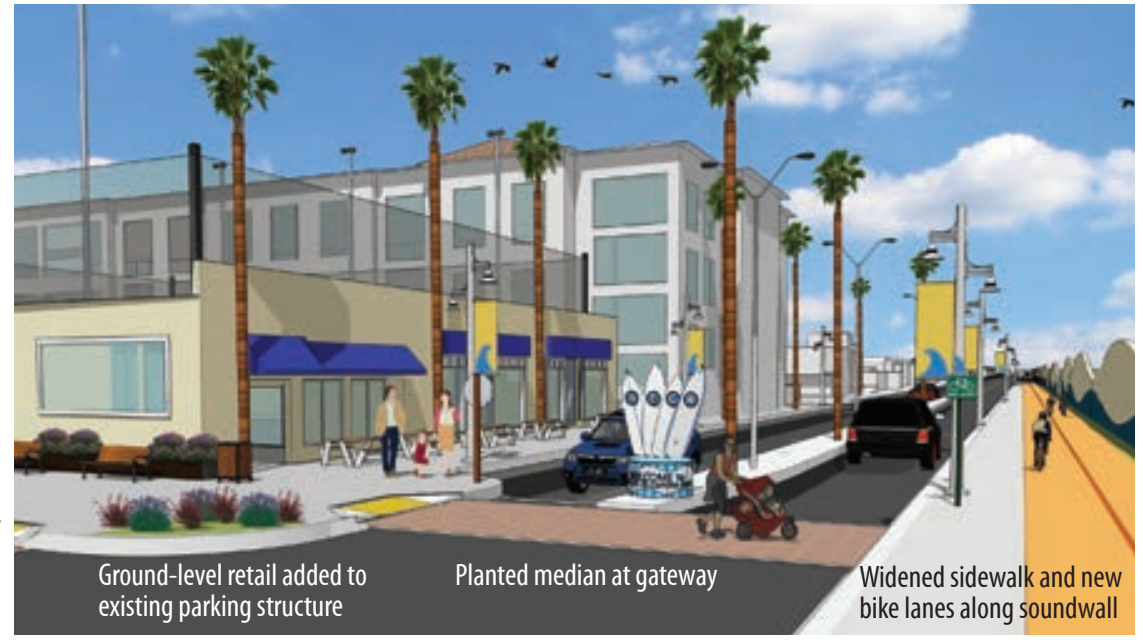

corridor that reflects the culture and character of the community and enhances the quality of life in the neighborhood."

A total of 16 development objectives are proposed, two for each urban design principle, and the design ideas were grouped into three main categories: Connections, Anchors, and Design Guidelines. The Connections concerns proposals for street design, parking, streetscaping (landscaping, street furniture, lighting, public art), connectivity elements, and access to parks, trails, and open space. SECR's roadway redesign includes comfortable sidewalks, pedestrian crossings and amenities, angled parking, a Class 1 bike lane, a redesigned sound wall, new street and pedestrian lighting, parklets and attractive landscaping. The pedestrian bridge will be renovated to make it safer, aesthetically attractive, and serve as a gateway into San Clemente for drivers on I-5. New pedestrian connections to San Luis Rey Park and from the Park to the golf facilities will increase connectivity, including through a weekly Farmers Market proposed for the park's parking lot. The alleyway running parallel to SECR will be transformed into a series of "woonerfs", or pedestrian community oriented spaces providing safe play spaces for children and for the community to socialize.

ing environment for people walking, biking, driving, or using transit. South El Camino Real will be an inviting and walkable

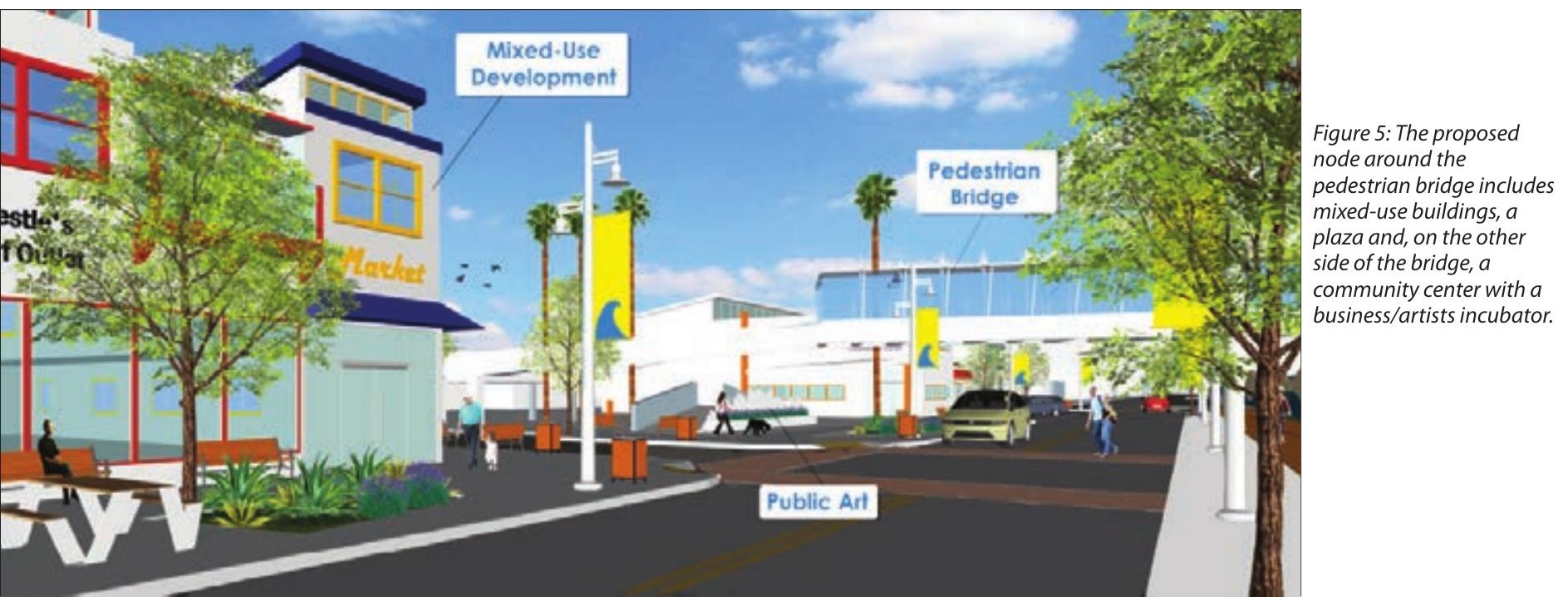


Proposals for Anchors highlight three nodes along the corridor with the potential to act as catalysts for the community, to attract new development, and to add to SECR's new identity. One will be located at the intersections with Avenida Magdalena, the main access to San Clemente's public golf course, and act as the North Gateway where retail will be encouraged at street level, pedestrian crossings will be provided, and a planted median will feature a landmark sculpture. In the middle of the corridor at the intersection with Avenida San Dimas and around the access to the pedestrian bridge, the Community Core will feature mixed use buildings, a community building with a business incubator and artists' lofts, and a small parklet; the redesigned bridge will be a strong marker in this node. The third node, the South Gateway next to the Trestles trailhead, will consist of a new surf-oriented retail complex next to the existing Carl's Jr, a small park with facilities for surfers, a public parking structure, a small bus terminal, and another landmark sculpture.

The third category, Design Guidelines, includes proposals for rezoning along SECR to encourage, in some areas, vertical mixed-use and street-oriented retail, while in others higher density residential. Site design guidelines encourage streetoriented buildings, and architectural guidelines control signage and, while embracing the diverse and eclectic nature of the existing structures, provide for more consistent and aesthetically-pleasing buildings along the corridor.

In the last chapter, the South El Camino Real Urban Design Concept Plan discusses the implementation process. An "Implementation Matrix" considers the degree that the various specific project proposals meet the eight urban design principles and their objectives, as well as their estimated time for full implementation. The matrix is meant to help city planners and community members to understand the relative importance of each proposal and their time frame, helping to prioritize actions. The funding possibilities more readily available for the projects implementation are in-kind developer contributions, city funds, and eight different state and federal grants.

\section{Concluding Remarks}

The limitations of this academic work are obvious from the short time span available for such a complex project to the limited involvement of the public and other city departments, such as public works and transportation. Nevertheless, the South El Camino Real Urban Design Concept Plan brings together a sound site and community assessment, a thorough theoretical foundation, and a collection of feasible, articulate and community oriented design concepts that are consistent with San Clemente's Centennial Plan.

Figure $6(a \& b)$ : The existing pedestrian bridge would be renovated to become safer and more aesthetically pleasing, serving as a gateway to San Clemente.

Figure 7: The node around the pedestrian bridge as seen from the south, showing the new undulating sound wall and the community center with a business/artists incubator.
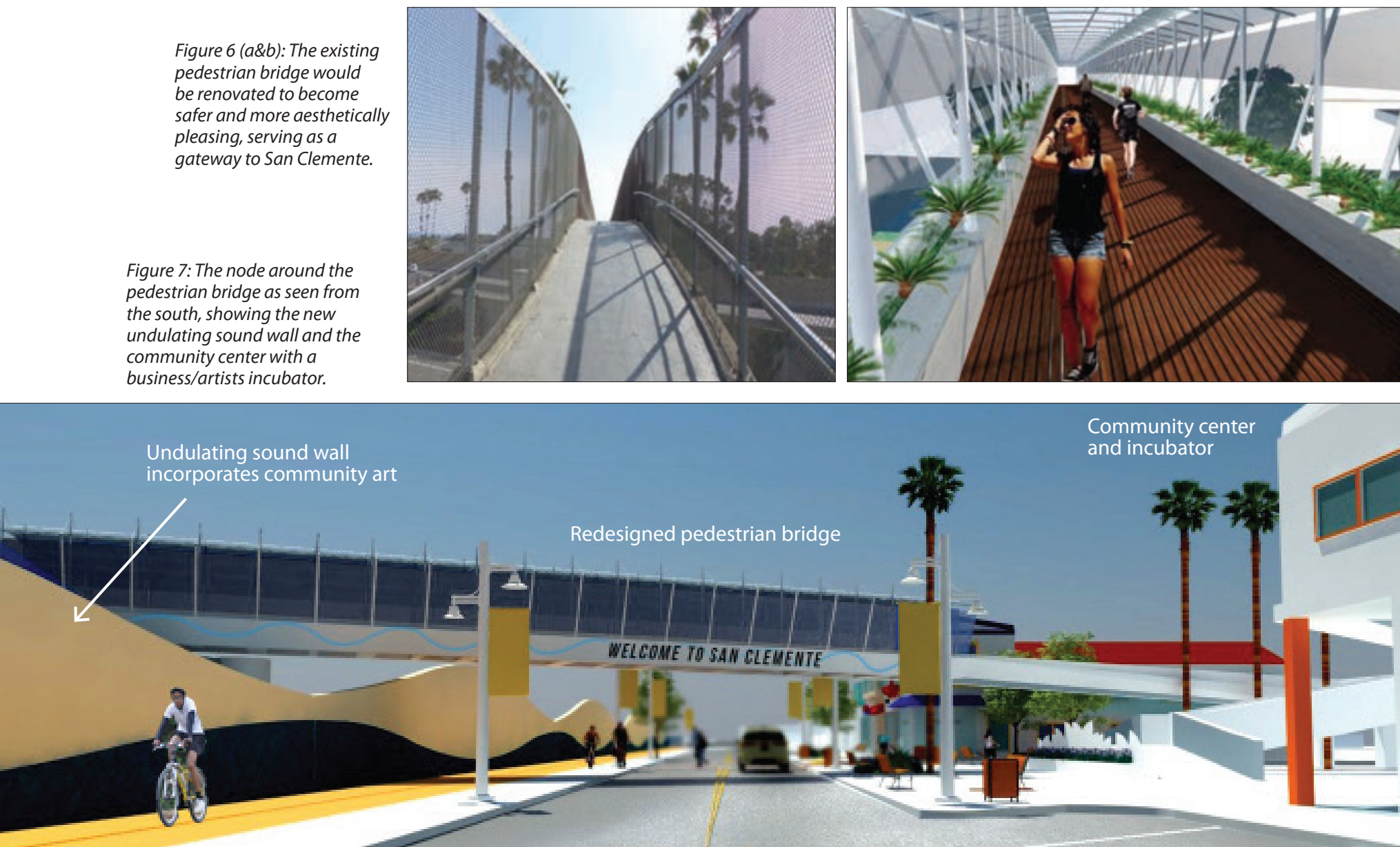


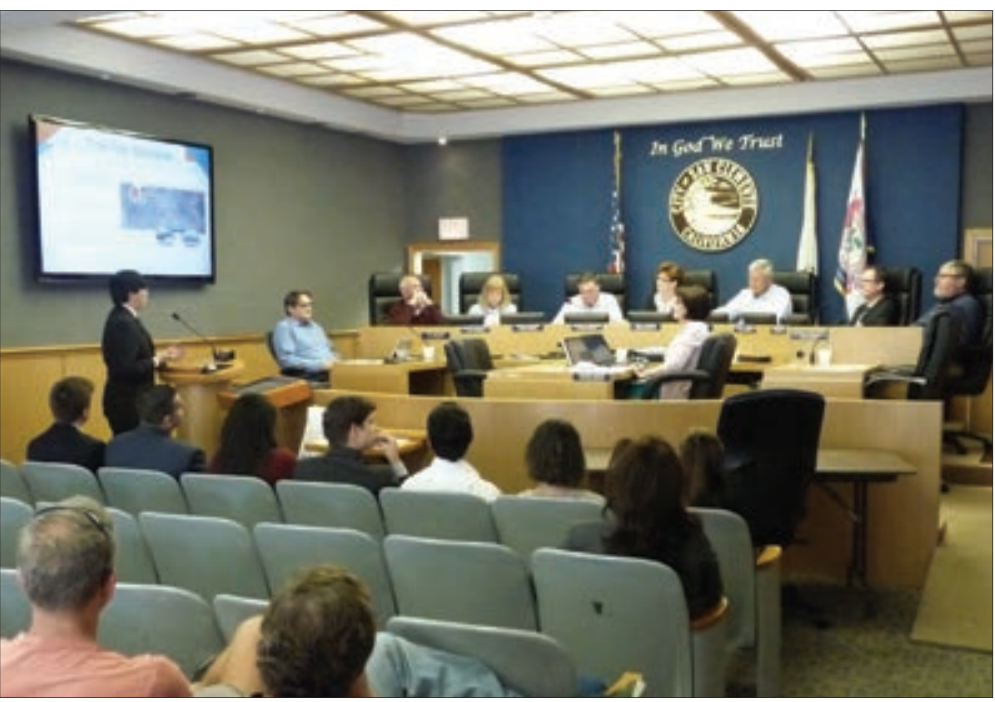

Figure 8: The class presenting the final proposals to the San Clemente City Council on June 3, 2015.

Our work responded to the city's intentions in hiring our studio: proposed concepts and ideas on how the corridor could become more visually appealing, economically attractive, and inviting for pedestrians and bicyclists. The final results and proposals were very positively received during the class presentations to San Clemente's Planning Commission and City Council at the end of the quarter. From an academic and pedagogical perspective, this project was both inspiring and very effective. We are grateful to the City of San Clemente and its Planning Division staff for the opportunity, and we sincerely hope that our work, concepts and suggestions may help in the future development of a sustainable, attractive, and community-oriented South El Camino Real corridor.

\section{References}

Barnett, Jonathan. 2003. Redesigning Cities: Principles, Practice, Implementation. Chicago: APA Press.

City of San Clemente. 2014. San Clemente's Centennial General Plan. Available at <http://san-clemente.org/departmentservices/planning-services/general-plan>.

City of San Francisco. 2010. Better Streets Plan. City of San Francisco Planning Department.

Ewing, Reid et Bartholomew, Keith. 2013. Pedestrian and Transit-Oriented Design. Washington, DC: Urban Land Insitute / American Planning Association.

Ewing, Reid et al. 2006. Measuring Urban Design Qualities - A Field Manual. Active Living Research. The Robert Johnson Foundation.

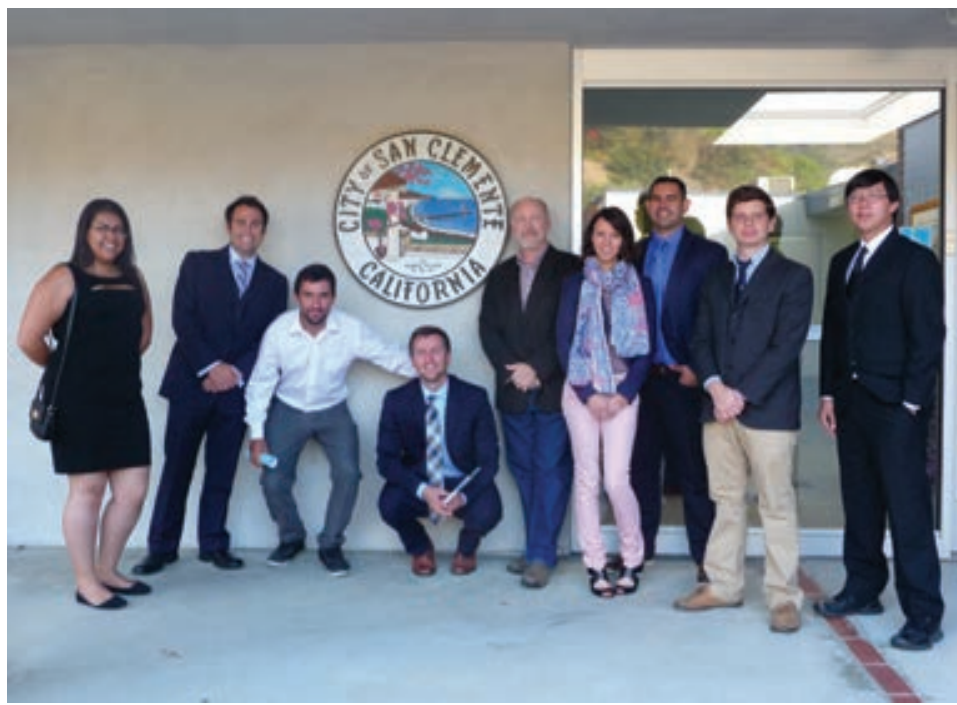

Figure 9: Part of the class posing with San Clemente planner Sean Nicholas (second from the left) outside the San Clemente's City Council building.

ITE - Institute of Transportation Engineers. 2006. Context Sensitive Solutions in Designing Major Urban Thoroughfares for Walkable Communities. Washington DC: Institute of Transportation Engineers.

Los Angeles County. 2011. Model Design Manual for Complete Streets. Department of Health and Human Services / Los Angeles County Department of Public Health / UCLA Luskin Center for Innovation.

MacCann, Barbara \& Suzanne Rynne (eds). 2010. Complete Streets: Best Policy and Implementation Practices. Chicago: American Planning Association.

NACTO - National Association of City Transportation Officials. 2013. Urban Street Design Guide. Washington DC: National Association of City Transportation Officials. 